\title{
DETECCIÓN DE PÉRDIDAS EN TUBERÍAS DE AGUA: PROPUESTA BASADA EN UN BANCO DE FILTROS
}

\section{LEAK DETECTION IN WATER PIPELINES: PROPOSAL BASED ON A BANK OF FILTERS}

\author{
Lucía Castro Burgos $^{1}$ Héctor Valdés-González ${ }^{2}$
}

Recibido 29 de febrero de 2008, aceptado 4 de septiembre de 2009

Received: February 29, 2008 Accepted: September 4, 2009

\begin{abstract}
RESUMEN
Actualmente la detección de pérdidas (DP) en tuberías de agua es un área de investigación activa, y con un creciente interés, debido a la importancia del transporte seguro del vital elemento. Este trabajo considera el problema de DP en tuberías de agua por medio de redundancia analítica, basada en un modelo matemático y utilizando técnicas de estimación de estados, teniendo por objetivo principal estudiar, proponer, implementar y aplicar algoritmos eficientes que permitan abordar de manera general el problema de DP. Para lograr esto se propone y evalúa un banco de filtros, implementados con filtros de Kalman (FK) y filtros de partículas (FP). Con esto se busca contribuir conceptualmente a la formulación del problema de DP, de manera modular, permitiendo que en un estudio futuro otras técnicas puedan ser utilizadas para resolver el problema. Además, obtener algoritmos eficientes y confiables, basados en un estimador de estados capaces de responder a requerimientos industriales, tales como: entregar, a partir de medidas de entrada y salida disponibles, una estimación fiable del estado del proceso, esto con independencia de la dinámica lineal o no lineal, asimismo, ser de fácil manejo y fácil configuración. La simulación computacional y los resultados experimentales muestran la efectividad de combinar FP con FK, para el caso básico de dos pérdidas secuenciales en una tubería, presentando ventajas de rápida convergencia y reducción del error de estimación, factores importantes en el problema de DP en ductos hídricos.
\end{abstract}

Palabras clave: Detección de pérdidas, banco de filtros, filtro de Kalman, filtro de partículas.

\begin{abstract}
Currently leak detection $(L D)$ in water pipelines is an active area of research that is attracting increasing interest due to the importance of the safe transport of this vital resource. This work considers the problem of $L D$ in water pipes by means of analytical redundancy, based on a mathematical model and using state estimation techniques. The main aim of this work is to research, propose, implement and apply efficient algorithms that allow to tackle generally the LD. To achieve this, a bank of filters including Kalman Filters $(K F)$ and Particle Filter $(P F)$ is proposed and evaluated. Thus a conceptual contribution to the formulation of the LD problem is proposed in a modular way so that future studies of other techniques can solve the problem. In addition, efficient and reliable algorithms are developed, based on a state estimator capable of responding to industrial standards such as the delivery, from input and output measures available, of a reliable estimate of the state of the process and that is independent of the linear or non-linear dynamics and easy to handle and configure. The computer simulation and the experimental results show the effectiveness of combining KF with PF for the simple case of two sequential leaks in a pipe, presenting advantages such as rapid convergence and reducing the estimation error which are important factors in LD in water pipeline.
\end{abstract}

Keywords: Leak detection, bank of filters, Kalman filter, particle filter.

1 Escuela Universitaria de Ingeniería Industrial, Informática y de Sistemas. Universidad de Tarapacá. Av. 18 de Septiembre 2222. Campus Saucache, Arica, Chile. E-mail: 113castro@yahoo.es

2 Universidad Andrés Bello. Facultad de Ingeniería. Escuela de Industrias, Ingeniería en Automatización y Robótica. Av. República 239. Santiago, Chile. E-mail: hvaldes@unab.cl 


\section{INTRODUCCIÓN}

Actualmente existe una gran diversidad de procesos industriales que emplean grandes sistemas de tuberías, de las cuales podemos destacar el transporte de agua. En razón de ello, se han construido complejos sistemas de redes de tuberías, a gran escala, los que han crecido y se han automatizado cada vez más en los últimos años. La operación segura de estos sistemas es primordial debido a que se trata de un elemento vital y valioso. Sin embargo, y a pesar de todas las medidas de precaución que se tomen, siempre existe la posibilidad de pérdidas por diversos factores, tales como rajaduras en la pared de la tubería, defecto en las junturas, corrosión, desgaste, entre otras.

El diagnóstico de fallas, disciplina enmarcada dentro del control tolerante a fallas (CTF), busca prevenir que fallas simples se conviertan en serias, con el fin de incrementar la eficiencia en la seguridad y reducir los riegos ante fallas. Este método implica tareas de detección y localización de pérdidas. La detección de pérdidas (DP) en tuberías es una aplicación industrial importante dentro del CTF y ha sido un área de investigación activa en las últimas dos décadas [1-3, 7-9, 11-15].

En el campo de DP de fluidos (tanto líquidos como gases) en tuberías se han investigado muchos métodos y técnicas para solucionar el problema. Pasando de métodos simples y económicos hasta algunos más complejos y costosos, podemos mencionar: métodos basados en el modelo dinámico, monitoreo de flujo, monitoreo acústico, monitoreo óptico, métodos de muestreo, entre otros; por ejemplo en [7,8] se puede obtener información más acabada de éstos, y obtener la descripción de otros métodos.

Entre los métodos basados en el modelo dinámico, se encuentran los observadores de estado, que son frecuentemente empleados en la DP. Existe una gran variedad de observadores de estado, incluyendo observadores lineales, no lineal, extendidos, adaptativos, y otros [6, 10]. Este tema ha sido de gran interés, siendo abordado, desde hace ya varios años, con investigaciones como las de Benkherouf y Allidina, Billmann e Isermann, hasta llegar a técnicas más actuales como las de Liu y Verde $[1,2,7,11-15]$.

Los métodos basados en modelos dinámicos se sustentan en el modelo matemático del flujo del fluido dentro de la tubería. Usando este modelo, los parámetros del flujo son calculados en diferentes secciones de la tubería y éstos son medidos. Entonces, las pérdidas pueden ser detectadas por comparación de los parámetros medidos y los calculados. En [2] se usa un observador de estado no lineal y una técnica de correlación especial para detectar y localizar pérdidas. [1] presenta un modelo de tubería con una pérdida, y emplea el FKE para estimar los parámetros de falla. En [1, 2], debido a que el transporte de fluidos a altas presiones es de naturaleza no lineal, los modelos que utilizan difieren en cuanto a las simplificaciones que realizan. Algunas de estas simplificaciones consideran que el flujo es horizontal, que el fluido es isotérmico, la compresibilidad despreciable y que la velocidad del flujo es mucho menor que la del sonido. [10], además, en este trabajo considera las perturbaciones de ruido y el error en el modelo de la tubería.

En [3] se estudió por simulación el problema de estimación de detección y localización de pérdidas, considerando un modelo simplificado de una cañería. Para la estimación se empleó un observador dinámico y como detector el método de correlación cruzada. En [9] también trabajó mediante simulaciones y presentó un algoritmo, el cual calcula presiones de entrada y salida, utilizando un modelo muy simple del ducto sin pérdida, las que luego son comparadas con las presiones medidas sobre el ducto real, utilizando métodos basados en el seguimiento de una función sumatoria de productos de correlación cruzada.

En trabajos más recientes, [7] propone el uso de un filtro de partículas adaptativo para abordar la detección y localización de pérdidas de una tubería de gas, logrando estimar con exactitud y rapidez el tamaño de la falla y su localización.

Verde ha abordado continuamente el problema de la detección y localización de pérdidas múltiples, que ocurren en forma simultánea, utilizando el filtro de Kalman como observador [11-15].

Este trabajo aborda el problema de DP en tuberías de agua, por medio de redundancia analítica, basada en el modelo matemático y utilizando técnicas de estimación de estados propuestas por Liu [7] y Verde [11-13], teniendo por objetivo principal estudiar, proponer, implementar y aplicar algoritmos eficientes que permitan abordar de manera general la DP. Para lograr esto se propone y evalúa un banco de filtros, con filtros de Kalman (FK) y filtros de partículas (FP). Con esto se busca contribuir conceptualmente a la formulación del problema de DP, de manera modular, permitiendo el estudio futuro de otras técnicas para resolver el problema. Además, obtener algoritmos eficientes y confiables, de fácil manejo y fácil configuración. 


\section{MODELO MATEMÁTICO DE UNA TUBERÍA}

\section{Modelo físico}

Para desarrollar el modelo no lineal de la tubería nos basaremos en las ecuaciones planteadas por Billmann [2]. Para una sección de tubería que transporta un fluido (gas o líquido), el balance de masas y energías conduce típicamente a las siguientes ecuaciones:

$$
\begin{gathered}
\frac{\varepsilon A}{b^{2}} \frac{\partial p}{\partial t}+\frac{\partial Q}{\partial z}=0 \\
\frac{\partial Q}{A \partial t}+\frac{\partial p}{\partial z}=-\frac{f b^{2}}{2 D A^{2}} \frac{Q|Q|}{p}
\end{gathered}
$$

Con $p$ presión $\left[\mathrm{N} / \mathrm{m}^{2}\right], Q$ flujo $\left[\mathrm{m}^{3} / \mathrm{s}\right], z$ coordenada de longitud [m], $t$ coordenada de tiempo [s], $f$ coeficiente de fricción [a-dimensional], $D$ diámetro de la tubería [m], $b$ es la velocidad del sonido en el fluido $[\mathrm{m} / \mathrm{s}], A$ área de sección transversal $\left[\mathrm{m}^{2}\right]$ y $\partial$ operador de derivada parcial.

El sistema ha sido simplificado tomando las siguientes consideraciones:

- La velocidad del sonido en el fluido, $b$, es constante y pequeña en comparación a la velocidad del sonido en el aire.

- Los efectos elásticos de la tubería son despreciables.

- El flujo que se desplaza a través de la tubería se asume horizontal.

- Para disminuir los errores debido a simplificaciones, se agrega un factor correctivo $\varepsilon$ al balance de masas como en [3].

En lo que resta, se trabajará con la carga de presión $H$, también conocida como altura piezométrica, que es la presión dividida entre la densidad y la aceleración gravitacional. Así que, despreciando la viscosidad y los efectos de turbulencia en el flujo, asumiendo que los cambios de temperatura dentro de la tubería y los intercambios de calor con el ambiente también son despreciables, que el modelo de la tubería es unidimensional, y reordenando los términos en función de las coordenadas de tiempo y espacio, tenemos:

$$
\frac{b^{2}}{\varepsilon} \frac{\partial Q(z, t)}{\partial z}+g A \frac{\partial H(z, t)}{\partial t}=0
$$

$$
\frac{\partial Q(z, t)}{\partial t}+g A \frac{\partial H(z, t)}{\partial z}+\mu Q(z, t)|Q(z, t)|=0
$$

Donde: $H$, es la carga de presión [m], $g$ es la aceleración de gravedad $\left[\mathrm{m} / \mathrm{s}^{2}\right]$ y $\mu=f / 2 D A$.

El modelo compuesto por las ecuaciones diferenciales (3) y (4) forma un sistema de parámetros distribuidos (SPD) del tipo hiperbólico. Una pérdida en el punto $p_{i}$ de la tubería produce una discontinuidad en (3) y (4), y por ello se emplea una condición de cota $p_{i}$ en el sistema, asociado con el flujo de descarga [12].

$$
Q_{p i}=\lambda_{i} \sqrt{H\left(p_{i}, t\right)}
$$

Donde el parámetro $\lambda_{i}>0$ es función del área del orificio, el coeficiente de descarga y la gravedad. Entonces, si $n-1$ pérdidas son asumidas, el comportamiento del fluido es descrito por $n$ par de ecuaciones diferenciales parciales, con una condición de cota entre cada sección, sin pérdidas, de la forma:

$$
Q_{p i}^{-}=Q_{p i}^{+}+Q_{p i}
$$

Donde $Q_{p i}^{-}$y $Q_{p i}^{+}$denotan el flujo antes y después de la pérdida en la posición $p_{i}$, respectivamente, para $i=1,2, \ldots, n-1$. Asumiendo que el flujo y presión en los extremos pueden ser medidos, las diversas combinaciones de condiciones de cotas completan la descripción del fluido. En este caso, la carga de presión en los extremos del ducto es seleccionada como condición de cota.

$$
[H(t, 0) \quad H(t, L)]
$$

Considerando que puede ser regulada con un controlador, y que el flujo de entrada y salida es definido como salida del sistema:

$$
[Q(t, 0) \quad Q(t, L)]
$$

\section{Modelo discreto}

El modelo de la tubería descrito en la sección anterior es un SPD. Para poder hacer uso del FK y FP, con el fin de estimar la pérdida $p_{i}$, se requiere de un modelo discretizado. Para ello, lo primero es dividir la tubería en $N$ secciones, como muestra la Figura 1. 


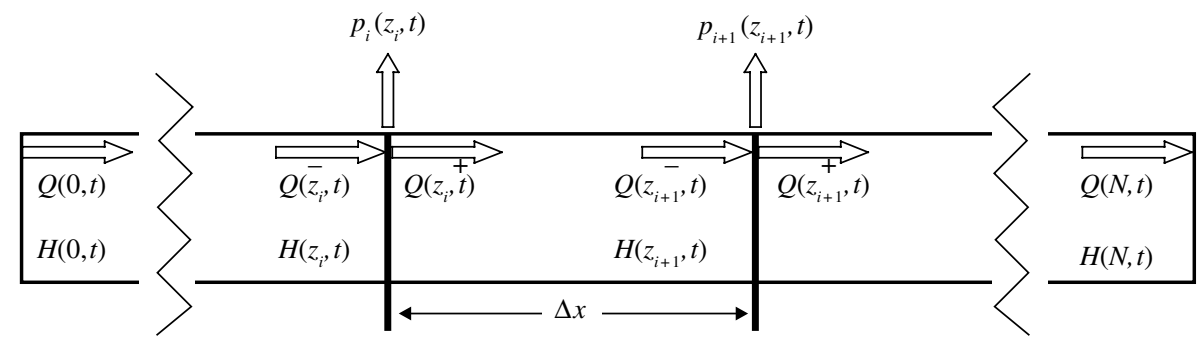

Figura 1. Representación de la tubería por secciones.

En el problema de detección de pérdidas, la condición de cota (6) genera un nuevo componente, el cual debe ser considerado en el proceso de discretización para obtener un modelo adecuado. Utilizaremos las aproximaciones (9) y (10) para derivar el modelo discreto. Asumiendo una tubería de largo $L$ donde:

- El espacio $z$ es dividido en $n$ celdas uniformes de tamaño $\Delta=L / n$, como muestra la Figura 1 .

- Las pérdidas están localizadas en el punto $p_{j}=\Delta \sum_{i=1}^{j} n_{i} \operatorname{con} n_{i}$ enteros para $j=1, \ldots, n-1$,

- Cada flujo de pérdida es caracterizado por el parámetro $\lambda_{j,} \mathrm{y}$

- Las derivadas parciales con respecto a $z$ en las ecuaciones (3) y (4) pueden ser aproximadas por:

$$
\begin{array}{cc}
\frac{\partial H(z, t)}{\partial z} \approx \frac{H_{i+1}(t)-H_{i}(t)}{\Delta} & \forall i=1, \ldots, n-1 \\
\frac{\partial Q(z, t)}{\partial z} \approx \frac{Q_{i}(t)-Q_{i-1}(t)}{\Delta} & \forall i=2, \ldots, n
\end{array}
$$

Donde el subíndice $i$ es asociado a la variable de la sección $i$.

Entonces los parámetros obtenidos en el modelo pueden ser escritos como $n$ pares de ecuaciones dinámicas no lineal acopladas, dadas por:

$$
\begin{aligned}
& \dot{Q}_{i}=a_{1} / \Delta\left(H_{i}-H_{i+1}\right)-\mu Q_{i}\left|Q_{i}\right| \quad \forall i=1,2 \ldots, n \\
& \dot{H}_{i}=a_{2} / \Delta\left(Q_{i-1}-Q_{i}-u_{t i} \lambda_{i} \sqrt{H_{i}}\right) \quad \forall i=2, \ldots, n
\end{aligned}
$$

Con constantes $a_{1}=g A, a_{2}=b^{2} / g A$ y $u_{t i}:=u\left(t-t_{i}\right)$, función asociada con la ocurrencia del tiempo $t_{i}$ de la pérdida $i$. Donde $H_{1}=H(t, 0)$ y $H_{\mathrm{n}+1}=H(t, L)$ son las entradas del sistema y obviaremos la variable independiente $t$ para simplificar la notación. Así el sistema (11) y (12) tiene una dimensión mínima de $m=2 n-1$.
Con pérdidas arbitrarias, caracterizadas por:

$$
\left(p_{k}, \lambda_{k}, t_{k}\right): \quad p_{k}=\Delta\left(n_{1}+\cdots+n_{. k}\right) \quad \forall k=1 \ldots, l
$$

Donde $l$ es el número total de pérdidas, con $\lambda_{k}>0, t_{k} \geq 0$.

Finalmente tenemos que el modelo no lineal (11) y (12) de dimensión $m$ puede ser escrito de forma vectorial como en (14). Con $n_{1,2}=n_{1}+n_{2}$, en el caso de dos pérdidas, flujo $Q_{i}$ y carga de presión $H_{i}$. Donde la sección de tamaño $\Delta$, de la discretización del espacio, debe satisfacer que:

$\sum_{i=0}^{l+1} n_{i} \Delta=n \Delta=L$, donde $l$ es el número de pérdidas.

$$
\left[\begin{array}{c}
\dot{Q}_{1} \\
\dot{H}_{2} \\
\dot{Q}_{2} \\
\vdots \\
\dot{H}_{n 1} \\
\vdots \\
\dot{H}_{n 1,2} \\
\vdots \\
\dot{Q}_{n-1} \\
\dot{H}_{n} \\
\dot{Q}_{n}
\end{array}\right]=\left[\begin{array}{c}
-\mu Q_{1}\left|Q_{1}\right|-a_{1} / \Delta\left(H_{2}-H_{1}\right) \\
a_{2} / \Delta\left(Q_{1}-Q_{2}\right) \\
-\mu Q_{2}\left|Q_{2}\right|+a_{1} / \Delta\left(H_{2}-H_{3}\right) \\
\vdots \\
a_{2} / \Delta\left(Q_{n 1-1}-Q_{n 1}-u_{t 1} \lambda_{1} \sqrt{H_{n 1}}\right) \\
\vdots \\
a_{2} / \Delta\left(Q_{n 1,2-1}-Q_{n 1,2}-u_{t 2} \lambda_{2} \sqrt{H_{n 1,2}}\right) \\
\vdots \\
-\mu Q_{n-1}\left|Q_{n-1}\right|+a_{1} / \Delta\left(H_{n-1}-H_{n}\right) \\
a_{2} / \Delta\left(Q_{n-1}-Q_{n}\right) \\
-\mu Q_{n}\left|Q_{n}\right|+a_{1} / \Delta\left(H_{n}-H_{n+1}\right)
\end{array}\right]
$$

\section{BANCO DE FILTROS}

\section{Filtro de Kalman extendido}

El filtro de Kalman extendido (FKE) es el principal algoritmo para estimar sistemas dinámicos representados 
en forma espacio estado, el que utilizando un modelo linealizado del proceso se emplea en procesos no lineal $[6,16]$.

Dado un proceso con un vector de estado $x \in \mathfrak{R}^{n}$, dominado por una ecuación diferencial estocástica no lineal, $x_{k}=f\left(x_{k-1}, u_{k}, w_{k-1}\right)$, con una medida $z \in \mathfrak{R}^{n}, z_{k}=h\left(x_{k}\right.$, $v_{k}$ ). Donde las variables aleatorias $w_{k} \mathrm{y} v_{k}$ representan el ruido del proceso y de la medida, respectivamente. La función no lineal $f$, relaciona el estado anterior en el paso $k$-1 con el estado actual en el paso $k$. Esta incluye como parámetros cualquier función que contenga una entrada $u_{k}$ y el ruido del proceso, de medida cero, $w_{k}$. La función no lineal $h$, relaciona el estado $x_{k}$ a la medida $z_{k}$.

Para estimar un proceso con relaciones no lineal, las ecuaciones que linealizan una estimación son:

$x_{k} \approx \tilde{x}_{k}+A\left(x_{k-1}-\hat{x}_{k-1}\right)+W w_{k-1}$,
$z_{k} \approx \tilde{z}_{k}+H\left(x_{k}-\tilde{x}_{k}\right)+V v_{k}$

$z_{k} \approx \tilde{z}_{k}+H\left(x_{k}-\tilde{x}_{k}\right)+V v_{k}$
Donde: $x_{k}$ y $z_{k}$ son el vector de estado y el vector de medida, actuales, $\tilde{x}_{k}$ y $\tilde{z}_{k}$ son la aproximación del vector de estado y del vector de medidas, $\tilde{x}_{k}$ es una estimación del estado, posterior, en el paso $k, w_{k} \mathrm{y} v_{k}$, son las variables aleatorias que representan el ruido del proceso y de la medida, respectivamente, con $A, W, H$ y $V$ matrices jacobianas.

El FKE funciona por medio de un algoritmo de predicción-corrección al pronosticar el nuevo estado, y su incertidumbre, y corregir la predicción con la nueva medida. El primer paso, la "predicción", consiste en generar un pronóstico del estado hacia adelante en el tiempo, tomando en cuenta toda la información disponible en ese momento y en un segundo paso, la "corrección”, se genera un pronóstico mejorado del estado, de tal manera que el error es minimizado estadísticamente. La Figura 2 muestra el funcionamiento básico del filtro comentado anteriormente.

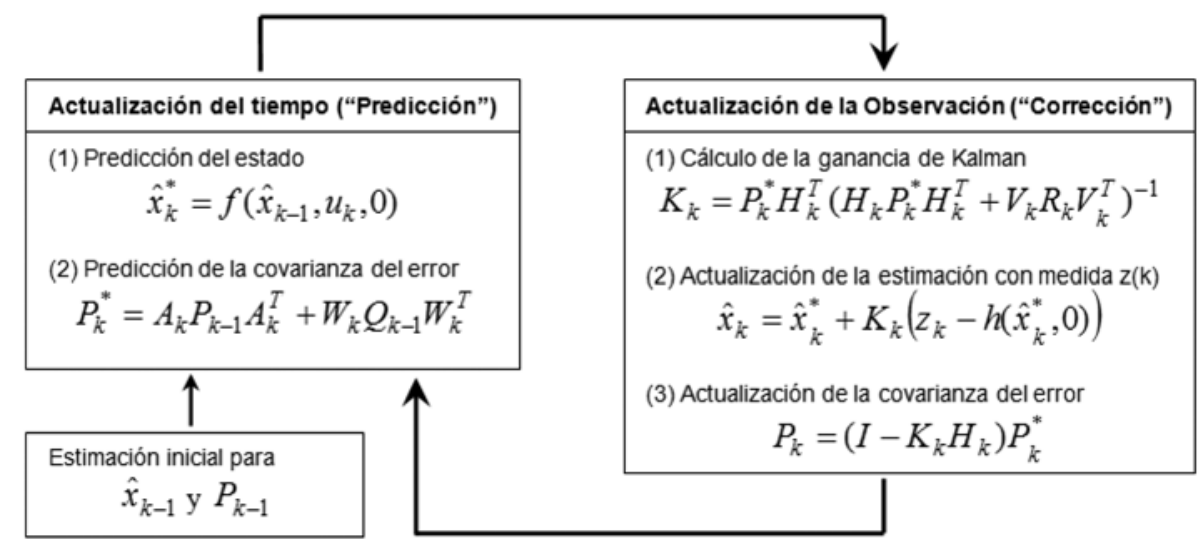

Figura 2. Esquema básico del funcionamiento del FKE.

Donde $Q$ y $R$ son las matrices de covarianza del error del proceso y la medida, respectivamente, $K$ es la matriz de ganancia de Kalman y $P$ es la matriz de covarianza del error de la estimación.

\section{Filtro de Kalman unscented}

El filtro de Kalman unscented (FKU) es una nueva herramienta de estimación introducida por Julier y Uhlmann [5], y luego perfeccionada por Wan y Van Der Merwe [17] para reemplazar al FKE en problemas de filtros no lineal, buscando superar inconvenientes tales como: Inestabilidad debido a la linealización y parámetros erróneos, costo en el cálculo de matrices jacobianas e irregularidad en la estimación. La ventaja principal es que no utiliza ninguna linealización para calcular la predicción de estados y covarianzas, lo que permite obtener una mejor estimación.

El FKU resuelve el problema de predicción probando la distribución de estados de una manera determinística, y entonces transformando cada una de las muestras por medio de la ecuación de transición de estados no lineal. Este proceso se conoce como transformación unscented. Para ver más detalles, de la TU y del FKU, revisar [17, 18].

El algoritmo básico del FKU puede definirse como sigue: 


\section{Paso 1: Iniciación}

$\hat{x}_{k+1 \mid k+1}=\hat{x}_{k+1 \mid k}+K_{k+1} V_{k+1}$

$P_{k+1 \mid k+1}=P_{k+1 \mid k}-K_{k+1} P_{k+1 \mid k}^{V} K_{k+1}^{T}$

Donde los vectores $\hat{x}_{k+1 \mid k}$ (predicción de estado), $V_{k+1}$ (innovación) y las matrices $K_{k+1}$ (ganancia de Kalman), $P_{k+1 \mid k}$ (covarianza de la predicción de estados) y $P_{k+1 \mid k}^{V}$ (covarianza de la innovación) son dependientes de las cantidades $\hat{x}_{k \mid k}$ y $P_{k \mid k}$.

Paso 2: Cálculo del punto sigma

$$
\begin{aligned}
& \sigma_{i+n}(k \mid k) \cong \hat{x}_{k \mid k} \pm\left(\sqrt{(n+k)\left(P_{k \mid k}+Q\right)}\right)_{i} \\
& W_{i+n} \cong 1 / 2(n+k) \\
& P_{k+1 \mid k}=\sum_{i=0}^{2 n} W_{i}\left[\sigma_{i}(k+1 \mid k)-\hat{x}_{k+1 \mid k}\right] \times\left[\sigma_{i}(k+1 \mid k)-\hat{x}_{k+1 \mid k}\right]^{T}
\end{aligned}
$$

Paso 3: Ecuaciones de actualización (del tiempo y medida)

$$
\begin{aligned}
& \hat{Z}_{k+1 \mid k}=H \hat{x}_{k+1 \mid k} \\
& V_{k+1}=Z_{k+1}-\hat{Z}_{k+1 \mid k} \\
& P_{k+1 \mid k}^{V}=H P_{k+1 \mid k} H^{T}+R \\
& K_{k+1}=P_{k+1 \mid k}^{x z}\left(P_{k+1 \mid k}^{V}\right)^{-1} \\
& P_{k+1 \mid k}^{x z}=P_{k+1 \mid k} H^{T}
\end{aligned}
$$

Las cantidades $\hat{x}_{k+1 \mid k}$ y $P_{k+1 \mid k}$ son llamadas predicción de estados y covarianza de la predicción del estado, respectivamente. Éstas son importantes para el funcionamiento general del filtro.

\section{Filtro de partículas}

El filtro de partículas (FP) ha emergido como una herramienta útil para solucionar problemas de estimación de estados en sistemas dinámicos. Teniendo como ventaja el que no necesita la linealización del modelo, por lo que su desempeño en la estimación de parámetros es bastante bueno. Además presenta cualidades interesantes como son su naturaleza probabilística, estructura no lineal y no gaussiana, capacidad de estimar estados híbridos y la posibilidad de ajustar su requerimiento computacional para la capacidad disponible. El FP, que es un método basado en la técnica secuencial de Monte-Carlo, fue propuesto por Gordon, Salmond y Smith [4].
Básicamente, el FP se compone de un conjunto de muestras (partículas) y sus valores, o pesos, asociados a cada una de esas muestras. La idea fundamental es utilizar dichas muestras para representar la distribución del estado posterior, dada una secuencia de medidas obtenida por sensores. Cuando se tiene esta nueva información, las partículas son constantemente reasignadas para actualizar la estimación del estado del sistema.

La eficacia y exactitud del FP dependen, principalmente, de dos factores fundamentales: el número de partículas usadas para estimar la distribución posterior y la función de propagación empleada para reasignar estas partículas en cada iteración.

Consideraremos un tipo de sistema no lineal de tiempo discreto de la forma:

$$
\begin{aligned}
& x_{k+1}=f\left(x_{k}, u_{k}, \theta_{k}\right)+w_{k} \\
& y_{k+1}=h\left(x_{k+1}\right)+v_{k+1}
\end{aligned}
$$

Donde $x_{k} \in \mathfrak{R}^{n}$ es el estado, $u_{k} \in \mathfrak{R}^{p}$ representa la entrada, $y_{k} \in \Re^{m}$ representa la salida, $\theta_{k} \in \Re^{l}$ es el vector de parámetros de falla, $f: \mathfrak{R}^{p} \times \mathfrak{R}^{n} \rightarrow \mathfrak{R}^{n}$ es la función de transición de estados, y $h: \mathfrak{R}^{n} \rightarrow \mathfrak{R}^{m}$ es la función de medidas. El ruido del proceso $w_{k} \in \mathfrak{R}^{n}$ es de medida cero, e independiente del estado actual, del estado pasado y del ruido del proceso.

A continuación se presenta una adaptación del algoritmo del FP, propuesto en [7], y que es aplicado a la detección de fallas.

\section{Paso 1: Inicialización}

El vector de estado es aumentado con el parámetro de falla desconocido:

$$
z_{k}:=\left[\begin{array}{l}
x_{k} \\
\theta_{k}
\end{array}\right]
$$

Las funciones del sistema y de la medida, del sistema aumentado, son $f_{e}$ y $h_{e}$, respectivamente. Probar $N s$ partículas $\left\{z_{0}^{i}, i=1, \ldots, N_{s}\right\}$ de la Función de Densidad de Probabilidad (FDP) supuesta $p\left(z_{0}\right)$.

\section{Paso 2: Predicción}

Probar $N s$ valores $\left\{w_{k}^{\theta i}, i=1, \ldots, N_{s}\right\}$ y $\left\{w_{k}^{\theta i}, i=1, \ldots, N_{s}\right\}$ desde la FDP de $w_{k}$ y $w_{k}^{\theta}$, respectivamente. Aquí $w_{k}^{\theta}$ es el ruido artificial del proceso, este es ruido blanco, gaussiano y con media cero, la varianza se fija para ser invariante y pequeña. Entonces se calcula: 
$x_{k+1 \mid k}^{i}=f_{e}\left(x_{k}^{i}, u_{k}\right)+w_{k}^{i}$,

$\theta_{k+1 \mid k}^{i}=\theta_{k}^{i}+w_{k}^{\theta i}$,

$y_{k+1 \mid k}^{i}=h_{e}\left(z_{k+1 \mid k}^{i}\right)$

Paso 3: Actualización

$\tilde{p}\left(z_{k+1} \mid D_{k+1}\right)=\sum_{i=1}^{N s} w^{i} \delta\left(z_{k+1}-\tilde{z}_{k+1 \mid k}^{i}\right)$, donde $\delta$ es la función Dirac-delta.

Paso 4: Remuestreo ${ }^{3}$

Remuestreando independientemente $N s$ tiempos desde la anterior distribución discreta. Las partículas resultantes $\left\{z_{k+1}^{i}, i=1, \ldots, N_{s}\right\}$ satisfacen:

$\operatorname{Pr}\left\{z_{k+1}^{i}=\tilde{z}_{k+1 \mid k}^{j}\right\}=w^{j}, j=1, \ldots, N_{s}$.

Entonces la FDP se convierte en:

$p\left(z_{k+1} \mid D_{k+1}\right)=\frac{1}{N_{s}} \sum_{i=1}^{N s} \delta\left(z_{k+1}-z_{k+1}^{i}\right)$

Y se calcula:

$\hat{z}_{k+1}=E\left[z_{k+1}^{i}\right]=\frac{1}{N_{s}} \sum_{i=1}^{N s} z_{k+1}^{i}$

Paso 5: Los pasos de predicción, actualización y remuestreo son recursivamente aplicados en cada paso del tiempo $k$.

A menudo el FP es una alternativa al FKE con la ventaja de que con suficientes muestras estas se acercan a la estimación bayesiana óptima, así este puede ser más exacto que el FKE. Además ofrece otra gran ventaja, y es la posibilidad de mejorar el rendimiento del filtro de partículas, por medio de su combinación con otros filtros, como podemos ver en [18] y en el presente trabajo, en el que se combina, específicamente, con los filtros de Kalman extendido y unscented.

\section{RESULTADOS DE SIMULACIÓN}

Existe una teoría general para el diagnóstico de fallas en sistemas dinámicos, que permite la generación de síntomas de fallas llamados "residuos", los que se obtienen por software [2, 9-12].

\footnotetext{
3 El término remuestreo hace referencia a la idea de reutilizar los datos disponibles de una muestra.
}

Aquí la tarea de diagnóstico puede desarrollarse en dos pasos principales, la generación y la evaluación de residuos.

En la etapa de generación de residuos emplearemos un banco de filtros para predecir el valor de la salida del sistema. La etapa de evaluación de residuos se obtiene mediante la diferencia entre las salidas medidas del sistema y las salidas estimadas para el modelo de operación normal. La Figura 3 sintetiza lo antes dicho.

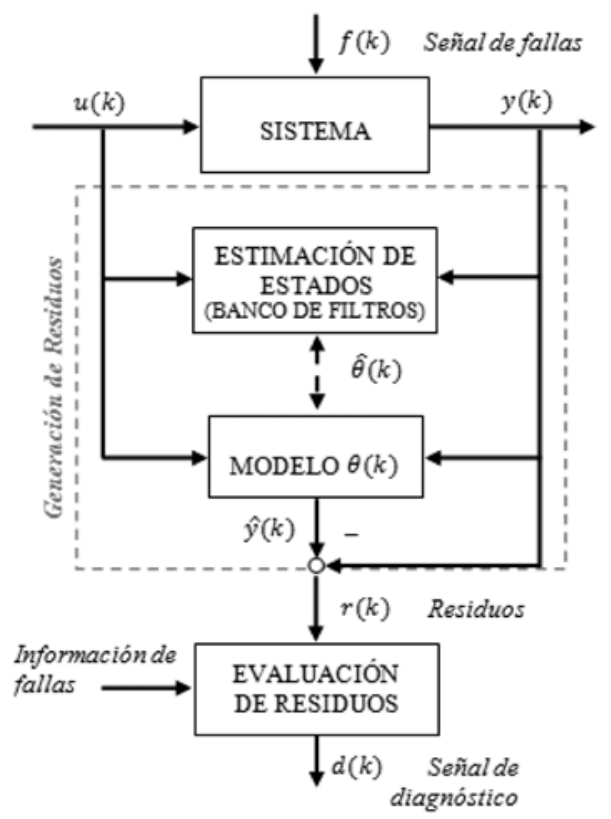

Figura 3. Esquema de DP mediante la estimación de estados.

En la simulación de la tubería de agua, hipotética, las condiciones iniciales son similares a las utilizadas en [15]. Se trabajó una tubería seccionada en 3 partes, de manera uniforme. La simulación se realizó en un periodo de muestreo $T=30[\mathrm{~min}]$, con intervalos de tiempo de $\Delta t=0.1$ [s], y un conjunto de 400 datos. Algunas de las condiciones iniciales y parámetros de la tubería, son:

$$
\begin{aligned}
& L=132.56 \mathrm{~m}, D=0.105 \mathrm{~m}, \text { espesor }=4.7 \mathrm{~mm}, \\
& f=0.04, b=1248 \mathrm{~m} / \mathrm{s}, a_{1}=0.0849 \mathrm{~m}^{3} / \mathrm{s}^{2}, \\
& \mu=21.99 \mathrm{~m}^{-3}, \lambda_{1}=6.2283 \times 10^{-5}, \lambda_{2}=1.0534 \times 10^{-4}, \\
& \Delta=41.18 \mathrm{~m}, z_{\text {eq }}=71.27 \mathrm{~m} .
\end{aligned}
$$

Dado $n=3$ secciones se producirá un modelo de orden $m=2 n-1=5$. Lo que produce, utilizando el modelo no lineal dado por (14), la siguiente matriz de ecuaciones de estado. 


$$
\left[\begin{array}{c}
\dot{Q}_{1} \\
\dot{H}_{2} \\
\dot{Q}_{2} \\
\dot{H}_{3} \\
\dot{Q}_{3}
\end{array}\right]=\left[\begin{array}{c}
-\mu Q_{1}^{2}-a_{1} / \Delta \times\left(H_{2}-u_{1}\right) \\
a_{2} / \Delta \times\left(Q_{1}-Q_{2}-\lambda_{1} \sqrt{H_{2}}\right) \\
-\mu Q_{2}{ }^{2}+a_{1} / \Delta \times\left(H_{2}-H_{3}\right) \\
a_{2} / \Delta \times\left(Q_{2}-Q_{3}-\lambda_{2} \sqrt{H_{3}}\right) \\
-\mu Q_{3}{ }^{2}+a_{1} / \Delta \times\left(H_{3}-u_{2}\right)
\end{array}\right],
$$

Con entrada: $u=\left[H_{1}, H_{4}\right]^{T}$ y salida $y=\left[Q_{1}, Q_{3}\right]^{T}$.

Tomando como entrada del filtro $u(t)$ y salida simulada del proceso $y(t)$, se realizó el proceso de estimación con cada filtro, a saber: Kalman extendido, Kalman unscented, partículas, y sus combinaciones FP-FKE y FP-FKU. En el FP se empleó un algoritmo de remuestreo sistemático y un número de 100 partículas.

Caso 1: una pérdida, con un $2 \%$ de ruido en la salida. El punto de fuga está dado en el instante $T=280$ [s], con un $2 \%$ de pérdida sobre el flujo de entrada. Con el fin de aproximar los datos a la realidad, se adicionó un ruido del $2 \%$ sobre la salida de la simulación.

De la Figura 4-a se puede notar que los filtros, aún ante la pérdida y el ruido presente, muestran una buena convergencia con la salida simulada, con bajos niveles de error, Figura 4-b.

Caso 2: dos pérdidas, con un 5\% de ruido en la salida. Se presentan dos puntos de pérdidas, la primera en el instante $T=100$ [s], con un $2 \%$ de pérdida sobre el flujo de entrada y la segunda, en $T=280$ [s], con un 5\% de pérdida. Además, se adicionó un 5\% de ruido sobre la salida de la simulación.

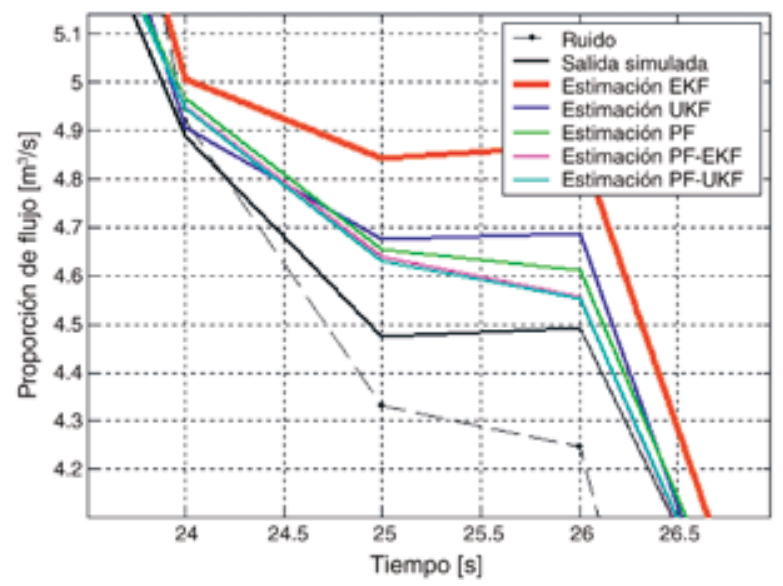

(a) Salida observada versus estimada de los cinco filtros (imagen ampliada).

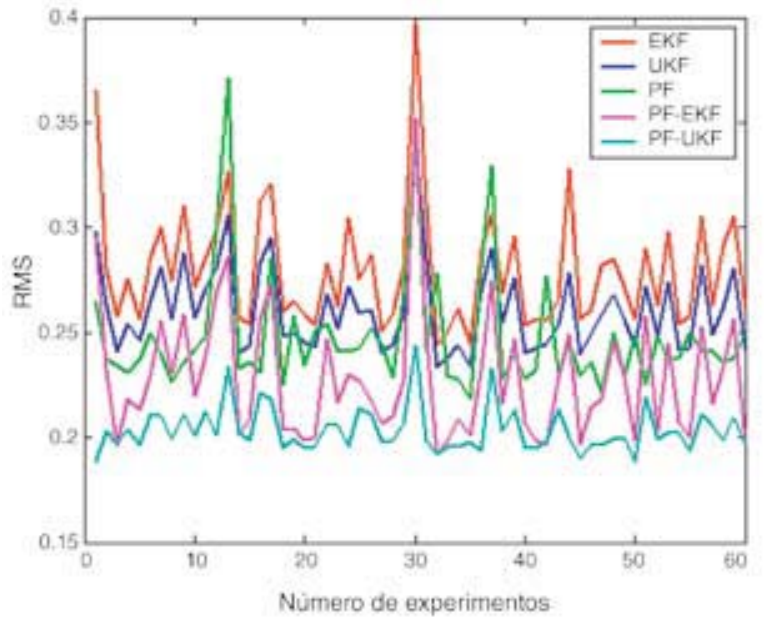

(b) Error RMS de los filtros.

Figura 4. Resultados de la simulación, en caso 1.

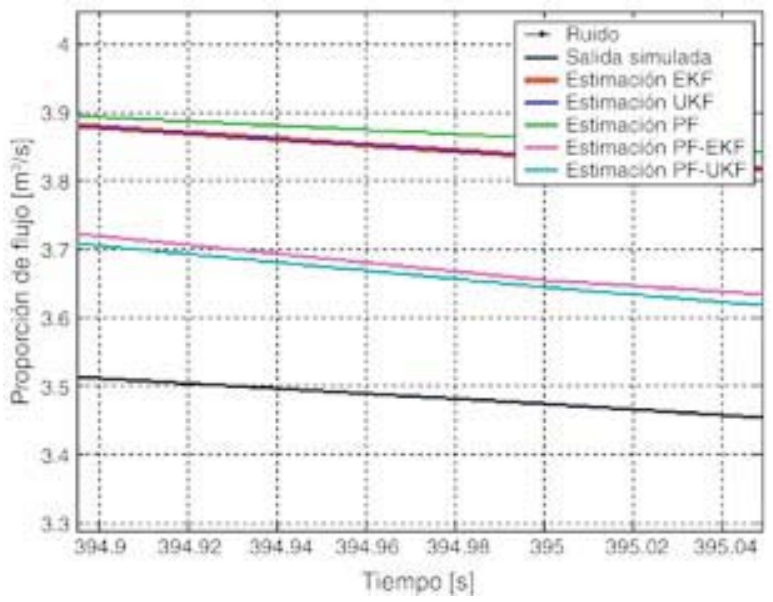

(a) Salida observada versus estimada de los filtros (imagen ampliada).

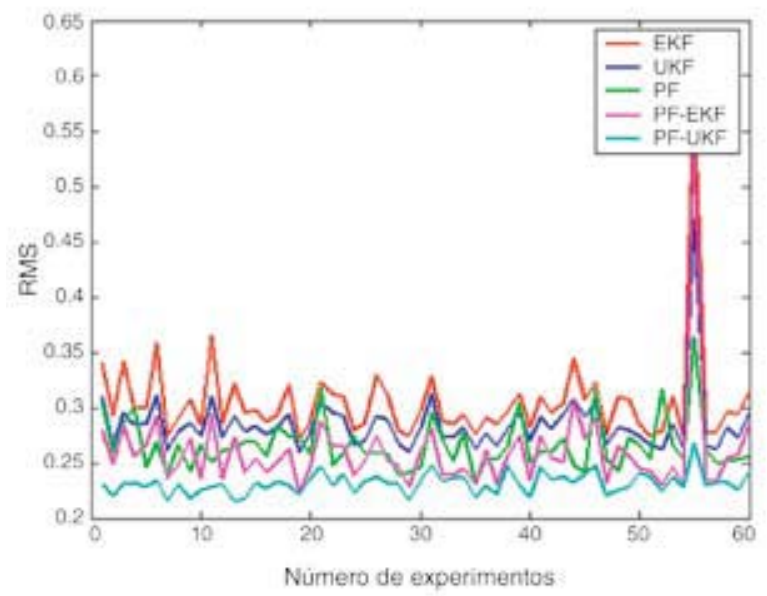

(b) Error RMS de los filtros.

Figura 5. Resultados de la simulación, en caso 2. 
En la Figura 5-a se puede apreciar que ante un leve aumento en el ruido, las curvas no varían de forma notoria, mostrando un comportamiento eficiente, ante una situación de pérdidas y ruido. También podemos percibir que el mejor desempeño en cuanto a precisión lo realiza el FP en sus tres modalidades. Y la Figura 5-b nos señala esto mediante el error RMS, destacando que la mejor precisión se tiene de la combinación FP-FKU.

En ambos casos podemos notar que el mejor filtro en esta aplicación es la combinación del FP con el FKU, dado que en todas las pruebas realizadas (incluyendo las no presentadas) y aún con condiciones de error más altas, como casos extremos, este filtro tiene una buena respuesta, alcanzando una buena y rápida convergencia hacia la curva real. Pero esto sólo es por simple observación, una métrica cuantitativa que nos indica con números lo antes dicho es el Error Cuadrático Medio (del inglés, RMS), que está definido por:

$$
R M S=\sqrt{\sum_{i=1}^{N} \frac{\left(\phi_{i}-\phi_{\text {iobs }}\right)^{2}}{N}}
$$

Donde $\phi_{i}$ es el valor estimado, $\phi_{i o b s}$ es el valor observado o simulado y $N$ es el número de valores analizados.

En la Tabla 1 podemos comparar exactitud vs tiempo de ejecución de cada algoritmo.

Tabla 1. Métricas de exactitud vs rapidez.

\begin{tabular}{|c|c|c|c|c|c|}
\hline \multirow{2}{*}{$\begin{array}{l}\mathfrak{Z} \\
\mathfrak{3}\end{array}$} & \multirow{2}{*}{$\%$ Ruido } & \multirow{2}{*}{ Filtro } & \multicolumn{2}{|c|}{ RMS } & \multirow{2}{*}{$\begin{array}{l}\text { Tiempo } \\
\text { Ejecución }\end{array}$} \\
\hline & & & Media & Varianza & \\
\hline$\cong$ & \multirow{5}{*}{$2 \%$} & FKE & 0.27995 & 0.00084397 & 1.2069 \\
\hline 5 & & FKU & 0.26068 & 0.0004249 & 1.3923 \\
\hline$\ddot{\overrightarrow{0}}$ & & FP & 0.24817 & 0.00081324 & 5.7747 \\
\hline$\tilde{u}^{3}$ & & FP-FKE & 0.22824 & 0.00090582 & 8.9409 \\
\hline & & FP-FKU & 0.20343 & 0.00011864 & 27.5049 \\
\hline \multirow{7}{*}{ 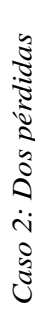 } & \multirow{2}{*}{$\%$ Ruido } & \multirow{2}{*}{ Filtro } & \multicolumn{2}{|c|}{ RMS } & Tiempo \\
\hline & & & Media & Varianza & Ejecución \\
\hline & \multirow{5}{*}{$5 \%$} & FKE & 0.30523 & 0.0018736 & 1.2602 \\
\hline & & FKU & 0.28482 & 0.00080124 & 1.4118 \\
\hline & & FP & 0.26602 & 0.00056061 & 5.4511 \\
\hline & & FP-FKE & 0.26011 & 0.0017031 & 8.7338 \\
\hline & & FP-FKU & 0.23164 & 0.00015809 & 27.7786 \\
\hline
\end{tabular}

De estas métricas cuantitativas podemos desprender lo siguiente: el FP es el filtro óptimo en cuanto a resultados; tanto por sí solo, como en combinación de filtros de Kalman, éste nos entrega la mejor y más rápida convergencia, es decir, se acerca rápidamente a la curva simulada, sin embargo, presenta el mayor tiempo de ejecución.

\section{Detección de pérdidas}

Se empleó el método de detección más simple que, como mencionamos, consiste en comparar los caudales de entrada y salida obtenidos durante el muestreo, esto es:

$$
Q_{\text {pérdida }}(k)=Q_{\text {salida }}(k)-Q_{\text {entrada }}(k)
$$

Existe pérdida si $Q_{\text {pérdida }}$ supera un valor umbral.

Una mejora notable se obtiene si se emplean los datos de los caudales filtrados, como:

$$
\begin{aligned}
& \Delta Q_{e}(k)=Q_{e}(k)-\hat{Q}_{e}(k) \\
& \Delta Q_{s}(k)=Q_{s}(k)-\hat{Q}_{s}(k) \\
& r(k)=\Delta Q_{e}(k)-\Delta Q_{s}(k)
\end{aligned}
$$

En caso de pérdida el caudal de entrada aumenta y el de salida disminuye, este comportamiento constituye lo que se conoce como firma de falla [9], por lo tanto:

$r(k)>$ umbral $>0 \Rightarrow$ falla

Siguiendo esta lógica, y tras varias pruebas realizadas, empleando un valor umbral de 2.5 , los resultados en cuanto al porcentaje de fallas detectadas por cada filtro, del total de casos analizados, son presentados en la Tabla 2.

Tabla 2. Porcentaje de fallas detectadas por los filtros.

\begin{tabular}{|c|c|c|c|c|c|c|}
\hline \multicolumn{2}{|c|}{} & FKE & FKU & FP & FP-FKE & FP-FKU \\
\hline \multirow{2}{*}{$\begin{array}{c}\% \text { Fallas } \\
\text { detectadas }\end{array}$} & Caso 1 & $85 \%$ & $100 \%$ & $95 \%$ & $90 \%$ & $100 \%$ \\
\cline { 2 - 7 } & Caso 2 & $95 \%$ & $100 \%$ & $100 \%$ & $95 \%$ & $100 \%$ \\
\hline
\end{tabular}

De los resultados obtenidos, en cuanto a los observadores se refiere, y tras las pruebas realizadas con pérdidas menores y mayores (desde 1\% hasta un $10 \%$ ), se puede concluir que el FP-FKU es el filtro más óptimo, de los aquí presentados, para detectar fallas, bajo las condiciones empleadas.

En el caso particular de la detección de pérdidas, se concluye que ocurrida la falla, las estimaciones proporcionadas por el FP siguen mucho mejor los niveles reales en relación a las estimaciones del FKE. Este mejor desempeño del FP se puede explicar por la capacidad de la distribución beta de asignar distintas varianzas a la estimación, con lo que es posible seguir la evolución de un parámetro que 
experimenta un cambio abrupto, sin aumentar el error de estimación del parámetro ni del estado.

Además, dado que no es necesaria la linealización en el modelo no lineal de la tubería, con el filtro de partículas el desempeño en la estimación de parámetros en buena. Siendo las principales ventajas del filtro las siguientes: el tamaño de la pérdida puede ser estimado con exactitud y con una rápida convergencia, el algoritmo es aplicable a ambos procesos de ruido gaussiano o no gaussiano, $y$ puede detectar, relativamente, pequeñas pérdidas.

Si bien, en forma comparativa, el FP-FKU tiene un tiempo de ejecución elevado, este tiempo es aceptable, puesto que a cambio nos entrega una rápida convergencia y un bajo error en la estimación, y como se dijo éstos son factores importantes en el problema de DP.

\section{CONCLUSIONES}

Una evaluación de herramientas clásicas ha sido presentada por contraste en este trabajo. En el que es posible observar un mejor desempeño del FKU, por sobre su símil FKE, además de destacar su fácil implementación computacional. Por su parte, el FP presenta cualidades interesantes como la posibilidad de ajustar su requerimiento computacional para la capacidad disponible, diseño simplificado y no necesita linealizar el modelo del proceso con el que se trabaja, entre otras características, que lo convierten en una buena opción para su aplicación al problema.

La simulación y los resultados experimentales obtenidos muestran que existe una mejor observación y desempeño de la estimación medida con el método de integración FP-FKU sobre el resto de los métodos, lo que permite detectar fallas de hasta un $2 \%$ del caudal en el $100 \%$ de los casos. Este filtro es altamente preciso y presenta características de rapidez y convergencia en condición no lineal, lo que permite obtener resultados óptimos, que es lo deseable en problemas de detección y diagnóstico de fallas. Además, dada la ventaja de poder combinar el FP con otros estimadores, en esta propuesta de banco de filtros, queda abierta la posibilidad para incorporar nuevos filtros o métodos de estimación, con el fin de obtener resultados adecuados según el tipo de proceso que se desee emplear.

\section{REFERENCIAS}

[1] Benkherouf and A.Y. Allidina. "Leak detection and location in gas pipelines". IEE Proc. Vol. 135, Pt. D, $\mathrm{N}^{\mathrm{o}} 2$, pp. 142-148. 1988.

[2] L. Billmann and R. Isermann. "Leak detection methods for pipelines". Automática. Vol. $23 \mathrm{~N}^{\circ} 3$, pp. 381-385. 1987.

[3] Cipriano y A. Medina. "Detección de pérdidas en sistemas de distribución de combustibles". Automática, Anales X Congreso de la Asociación Chilena de Control Automático, Facultad de Ingeniería, Pontificia Universidad Católica de Chile. Santiago, Chile. 1992.

[4] N.J. Gordon, D.J. Salmond and A.F.M. Smith. "Novel approach to nonlinear/non-Gaussian Bayesian state estimation". IEE Proc. F. Vol. 140 $\mathrm{N}^{\circ}$ 2, pp. 107-113. 1993.

[5] S. Julier and J. Uhlmann. "A new extension of the Kalman filter to nonlinear systems". Proc. of AeroSense: The 11th Int. Symp. on Aerospace/ Defense Sensing, Simulation and Controls. Orlando, FL. 1997.

[6] R.E. Kalman. "A new approach to linear filtering and prediction problems". Trans. ASME, J. Basic Engineering. Vol. 82, pp. 94-35. 1960. Fecha de consulta: Abril 2008. URL: http://www.cs.unc. edu/ welch/kalman/

[7] M. Liu, S. Zang and D. Zhou. "Fast leak detection and location of gas pipelines based on an adaptive particle filter". Int. J. Appl. Math. Comput. Sci. Vol. $15 \mathrm{~N}^{\circ} 4$, pp. 541-550. 2005.

[8] D. Misiunas. "Failure monitoring and asset condition assessment in water supply systems". Doctoral dissertation in industrial automation. Department of Industrial Electrical Engineering and Automation. Lund University. Lund. ISBN 91-88934-40-3. 2005.

[9] J.A. Motto y D. Marqués. "Detección y localización on-line de pérdidas en gasoductos". Instituto de Desarrollo Tecnológico para la Industria Química (INTEC). Santa Fe, Argentina. Automática, Tecnología para el Desarrollo. Vol. 1, Anales: III Congreso Latinoamericano de Automática, VIII Congreso de la Asociación Chilena de Control Automático. Viña del Mar, Chile. 1988. 
[10] D.N. Shields, S.A. Ashton and S. Daley. "Design of nonlinear observer for detecting faults in hydraulic sub-sea pipelines". Contr. Eng. Pract. Vol. $9 \mathrm{~N}^{\circ} 3$, pp. 297-311. 2001.

[11] C. Verde. "Multi-leak detection and isolation in fluid pipelines". Control Engineering Practice. Vol. 9, pp. 673-682. 2001.

[12] C. Verde. "Accommodation of multi-leak location in a pipeline". Contr. Eng. Pract., Vol. 13 N$^{\circ}$ 8, pp. 10711078. 2005.

[13] C. Verde and N. Visairo. "Identificability of multileaks in a pipeline". In Proceedings of the American control conference. ISBN-0-7803-8336-2. 2004.

[14] C. Verde and N. Visairo. "Multi-leak isolation in a pipeline by unsteady state test". 44th IEEE Conference on Decision and control and European control conference ECC. 2005.
[15] C. Verde, N. Visairo and S. Gentil. "Two leaks isolation in a pipeline by transient response". Advances in Water Resources. Vol. $30 \mathrm{~N}^{\mathrm{o}}$ 8, pp. 1711-1721. 2007.

[16] G. Welch and G. Bishop. "An introduction to the Kalman filter". Technical Report TR 95-041, Department of Computer Science. University of North Carolina at Chapel Hill. 1995.

[17] E.A. Wan and R. Van Der Merwe. "The unscented Kalman filter for nonlinear estimation". Proc. of IEEE Symposium (AS-SPCC). Lake Louise. Alberta. Canada. October 2000.

[18] E.A. Wan and R. Van Der Merwe. "Kalman filtering and neural networks". Wiley. Chapter 7: The Unscented Kalman Filter. 2001. Fecha de consulta: Mayo 2008. URL: http://citeseer.ist.psu. edu/wan01chapter.html 